\title{
Machen Studien gesund? - So hilft Forschung den Patienten
}

TESTS IN DER PRAXIS: Einleuchtende Theorien und Erkenntnisse „aus dem Labor“ müssen sich bewähren. Kurzsichtige Konsequenzen, die manche Kliniker bisweilen aus der aktuellen Studienlage ziehen, helfen im therapeutischen Alltag nicht weiter. Andere fragen sich: Kann ich die Erkenntnisse aus klinischen Studien auf mein klinisches Umfeld übertragen? Wie gehe ich mit widersprüchlichen Erscheinungen um?

Als der Autor vor Jahren einem Patienten von einer wissenschaftlichen Konferenz und den interessanten Forschungsergebnissen berichtete, fragte der Patient ihn frei heraus: „Machen mich Deine Studien gesund?“ Seine Antwort damals: „Die Chancen darauf steigen auf jeden Fall.“ Eigentlich hält er es aber mit Lukas Podolski: „Wichtig is' auf'm Platz!“

\section{Anamnese}

Eine 39-jährige Försterin stellt sich mit rezidivierenden unilateralen Rückenschmerzen rechts vor. Die Beschwerden bestehen seit fünf Jahren und strahlen zeitweise über das rechte Gesäß und den lateralen Oberschenkel bis zum Knie aus ( $\bullet$ Abb. 1). Die Beschwerden haben sich in den letzten Jahren kaum verändert. Es gab immer wieder Wochen oder Monate, in denen die Beschwerden kaum präsent waren. Dann traten sie wieder verstärkt auf. Die Patientin ist eine sehr aktive Sportlerin. Im Sommer fährt sie Rad und läuft regelmäßig. Im Winter macht sie nahezu täglich Skilanglauf ( Abb. 2). Intensives Lauftraining - vor allem Berglauf und lange Mountainbiketouren führen jedes Mal zu bleibender Verschlechterung. In den letzten Monaten stand der Knieschmerz im Vordergrund. Die Intensität des Gesäßschmerzes gibt die Patientin auf der Visuellen Analogskala (VAS) mit 6 an, die Oberschenkel- und Kniebeschwerden mit 4 (VAS).
Die Patientin hat bereits eine bunte Palette konservativer Therapien erlebt. Von Manueller Therapie für LWS und Hüfte über intensives Rumpfmuskeltraining bis hin zur kompletten Sportabstinenz. Keine Intervention brachte nennenswerte und anhaltende Verbesserungen. Aussicht auf Erfolg sieht sie eher nicht. Das Datum der ärztlichen Verordnung liegt fast fünf Monate zurück.

\section{Fragebogen - Einschränkung im Alltag}

Der Roland \& Morris Funktionsfragebogen zeigt mit 2/24 keine relevante Beeinträchtigung im Alltag. Standardisierte Fragebögen werden bisweilen der individuellen Situation von Patienten nicht gerecht. Patientenspezifische funktionelle Outcomes (PSFO) sind aussagekräftiger $[2,16]$ und sehr einfach in die Untersuchung einzubauen [6].
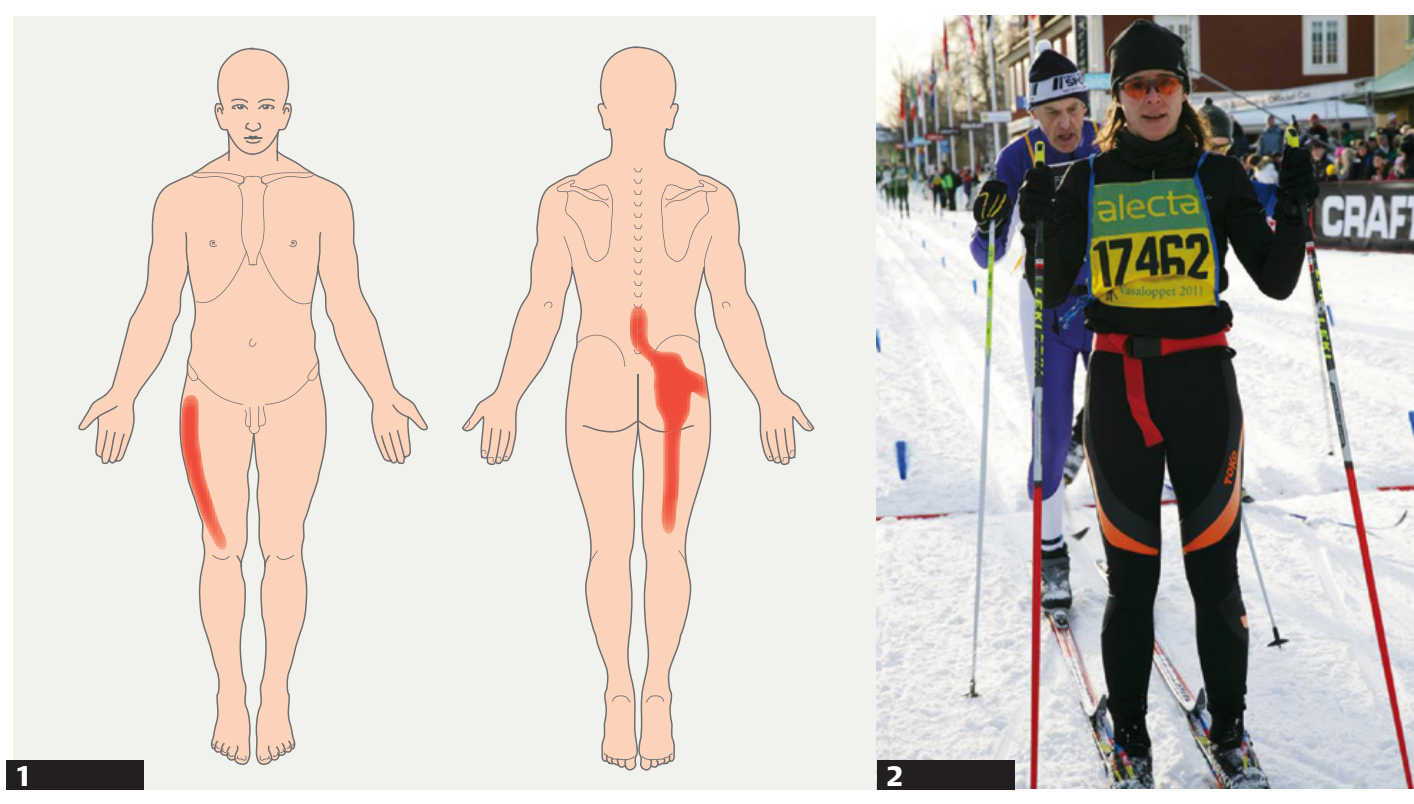

Abb. 1 Körperkarte der Patientin (Försterin, 39 Jahre).

Abb. 2 Die Patientin beim Skilanglauf. 
Die Antworten auf die Frage nach Aktivitäten im Alltag, die sie zurzeit nicht oder nur eingeschränkt ausführen kann, ergeben ein differenzierteres Bild. Die Patientin gibt drei Aktivitäten an.

Die patientenspezifischen funktionellen Outcomes am Behandlungstag 1:

1. Berglauf: 2;

2. Intensiv Mountainbikefahren: 2;

3. Schwere körperliche Arbeit - z. B. Harken bei der Gartenarbeit: 2.

Die Zahlen geben die persönliche Einschätzung an auf die Frage: Wie viel davon ist möglich? Nichts $=0$, wenig $=1$, äßig $=2$, viel $=3$, alles $=4$.

\section{Klinische Untersuchung}

Die klinische Untersuchung wurde nach dem Konzept der Mechanischen Diagnose und Therapie durchgeführt [15].

Die Beweglichkeit der Wirbelsäule ist unauffällig, ein Bewegungsverlust in keine Richtung zu beobachten.

Repetierte Testbewegungen der Lendenwirbelsäule zeigten kein klares mechanisches Muster ( Abb. 3-8). Sowohl repetierte Flexion im Stehen $(\checkmark$ Abb. 3 ) als auch Seitgleiten nach links $(\downarrow$ Abb. 5) produzieren endgradige Schmerzen im rechten Gesäß. Diese halten jedoch nach dem Beenden der Bewegungen nicht an. Repetierte Flexion in Rückenlage ( $\checkmark$ Abb. 7) produziert initial einen Schmerz im rechten Gesäß und Oberschenkel. Nach etwa 20
Wiederholungen hat die Patientin keine Schmerzen mehr. Die Bewegung scheint mit zunehmender Wiederholung etwas harmonischer zu sein. Im Anschluss wiederum durchgeführte Flexionsund Seitgleitbewegungen im Stehen haben jedoch den gleichen Effekt wie zuvor. Die Bewegung ist zunächst schmerzhaft und nach etwa 20 Wiederholungen schmerzfrei.

\section{Therapie \\ Besuch 1}

An dieser Stelle ist der nach Effektivität strebende Kliniker natürlich versucht, weitere potenzielle Schmerzgeneratoren, wie Hüftoder Sakroiliakalgelenk, zu untersuchen. Um falsch positive Befunde zu vermeiden, empfiehlt es sich aber, zuerst die Lendenwirbelsäule als Schmerzursache auszuschließen [9, 12, 15]. Oftmals wird das Bild klarer, wenn der Patient über längere Zeit den Effekt einer bestimmten Bewegungsrichtung evaluiert [17]. Der Therapeut wählt Flexion im Liegen als Probebehandlung über 48 Stunden. Die Patientin soll die Übung möglichst alle zwei Stunden zehn Mal durchführen. Flexion im Liegen empfiehlt sich, da diese Bewegung als einzige einen temporären Effekt auf die Mechanik zu haben scheint.

\section{Besuch 2}

Zwei Tage später stellt sich die Patientin wieder in der Praxis vor. Sie hat die Übungen regelmäßig durchgeführt. Von dem anamnestischen Wiederbefundzeichen hat sich keines verändert und auch

\section{TIPP FÜR DIE PRAXIS}

\section{Patienten-spezifische funktionelle Outcomes}

Bei der Befundaufnahme fragt der Therapeut: „Was sind drei Dinge in Ihrem Leben, die Sie wegen Ihren Schmerzen nicht oder nur eingeschränkt tun können und die Sie wieder tun möchten, wenn die Behandlung erfolgreich ist?"

Oder er fordert den Patienten auf: „Nennen Sie mir drei Aktivitäten in Ihrem Leben, die wegen Ihren Beschwerden nur eingeschränkt möglich sind und die Sie gerne wieder besser oder häufiger tun möchten.“

Beispiel: Patientenspezifische funktionelle Outcomes am Anfang der Behandlungsserie

\begin{tabular}{|l|l|l|l|l|l|}
\hline Aktivität & nichts & wenig & mäßig & viel & alles \\
\hline Punkte & 0 & 1 & 2 & 3 & 4 \\
\hline Aktivität 1 & $\mathrm{X}$ & & & & $>$ \\
\hline Aktivität 2 & & & $\mathrm{X}$ & & $>$ \\
\hline Aktivität 3 & & $\mathrm{X}$ & & & $\longrightarrow$ \\
\hline
\end{tabular}

Der Patient kann sich somit um 9 Punkte verbessern $(4+2+3)$; das heißt, wenn er sich um 9 Punkte verbessert, kann er wieder alle Aktivitäten vollständig ausüben.

Bei Zwischenerhebungen und/oder am Ende der Behandlungen stellt der Therapeut erneut die Frage zu den Einschränkungen.
Anschließend fragt der Therapeut: „Wie viel ist davon möglich?“ Der Patient erhält mehrere Auswahlmöglichkeiten für seine Antwort:

- nichts $=0$;
- wenig $=1$;
- mäßig = 2 ;
- viel = $3 ;$
- alles $=4$.

Beispiel: Patientenspezifische funktionelle Outcomes am Ende der Behandlungsserie

\begin{tabular}{|l|l|l|l|l|l|}
\hline Aktivität & nichts & wenig & mäßig & viel & alles \\
\hline Punkte & 0 & 1 & 2 & 3 & 4 \\
\hline Aktivität 1 & & & $>\mathrm{X}$ & & \\
\hline Aktivität 2 & & & & $>\mathrm{X}$ & \\
\hline Aktivität 3 & & & & & $>\mathrm{X}$ \\
\hline
\end{tabular}

Die erreichte Verbesserung beträgt 6 Punkte $(2+1+3)$ oder $67 \%$. 

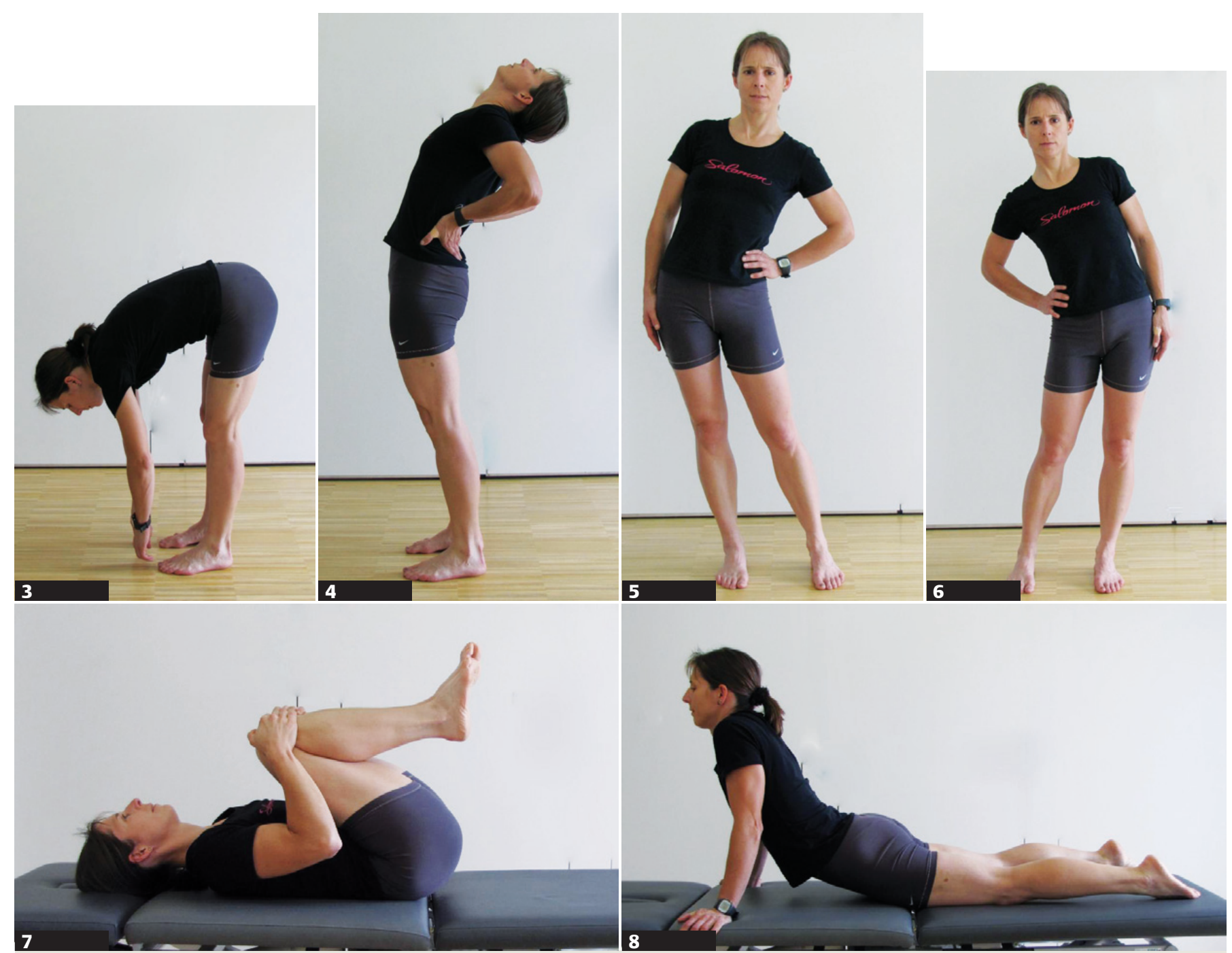

Abb. 3 Repetierte Flexion im Stehen.

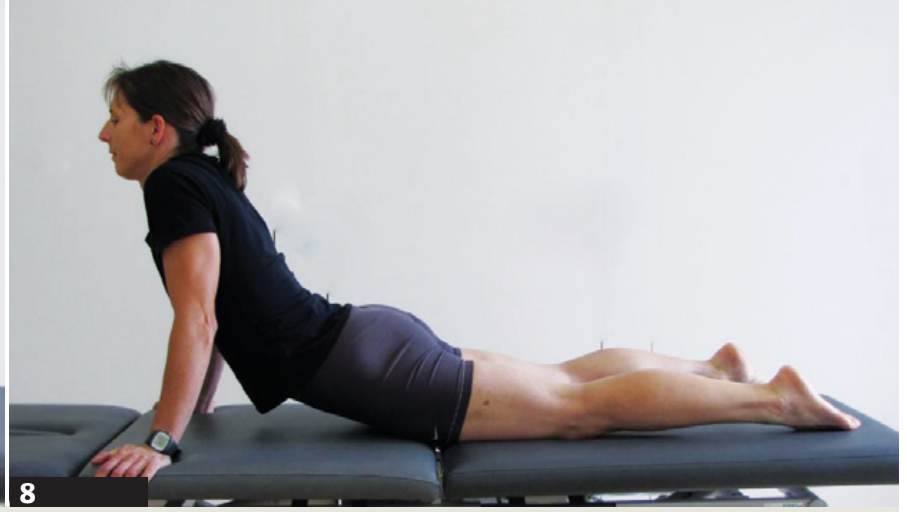

Abb. 4 Repetierte Extension im Stehen.

Abb. 6 Seitgleiten nach rechts.

Abb. 5 Seitgleiten nach links.

Abb. 7 Repetierte Flexion in Rückenlage.

Abb. 8 Repetierte Extension in Bauchlage.

die patientenspezifischen funktionellen Outcomes (PSFO) sind unverändert. Klinisch zeigt sich das gleiche Bild wie an Tag 1. Die Beweglichkeit der Lendenwirbelsäule ist in alle Richtungen frei. Endgradige Flexion im Stehen produziert nach wie vor einen Gesäßschmerz rechts. Genauso wie Seitgleiten nach links. Die Kontrolle der Eigenübung gibt keinen Anlass zur Korrektur.

Aktive und passive Untersuchung der Hüftgelenke zeigen keinerlei Auffälligkeiten.

Als mögliche Schmerzursache drängt sich nun das SIG auf. Schmerzlokalisation sowie die negative LWS- und Hüftuntersuchung sprechen dafür.

Die im Anschluss durchgeführten Provokationstests [10] sind jedoch allesamt negativ. Nichtsdestotrotz entschließt sich der Therapeut, zusätzlich eine Untersuchung des SIG mittels repetierten Testbewegungen durchzuführen [15]. 20 wiederholt ausgeführte Rotationen des rechten Iliums nach anterior $(\bullet$ Abb. 9a) zeigen während und nach den Tests keinerlei Effekt. Auch die zu-

sätzliche Applikation von Überdruck durch die Patientin ( $\bullet$ Abb. 9b) ändert nichts. Repetierte Rotation des Iliums nach posterior ( Abb. 10) zeigt nach zehn Wiederholungen keinen Effekt. Nach weiteren fünf Repetitionen gibt die Patientin einen langsam stärker werdenden Gesäßschmerz an, der jedoch nach weiteren zehn Bewegungen wieder verschwindet. Um den Effekt dieser Bewegungsserie auf die Wiederbefundzeichen zu evaluieren, führt die Patientin im Anschluss nochmals eine Flexionsbewegung im Stehen aus. Hierbei tritt kein Gesäßschmerz mehr auf. Auch wiederholte Flexionsbewegungen im Stehen lösen den Schmerz nicht mehr aus. Seitgleiten im Stehen war nun initial ebenso schmerzfrei möglich. Bei wiederholtem Seitgleiten stellt sich jedoch wieder ein leichter Gesäßschmerz ein. Dieser verschwindet aber sofort nach Beendigung der Bewegungen. Die Patientin beschreibt die getesteten Bewegungen nun als insgesamt flüssiger - ohne das näher erklären zu können. 

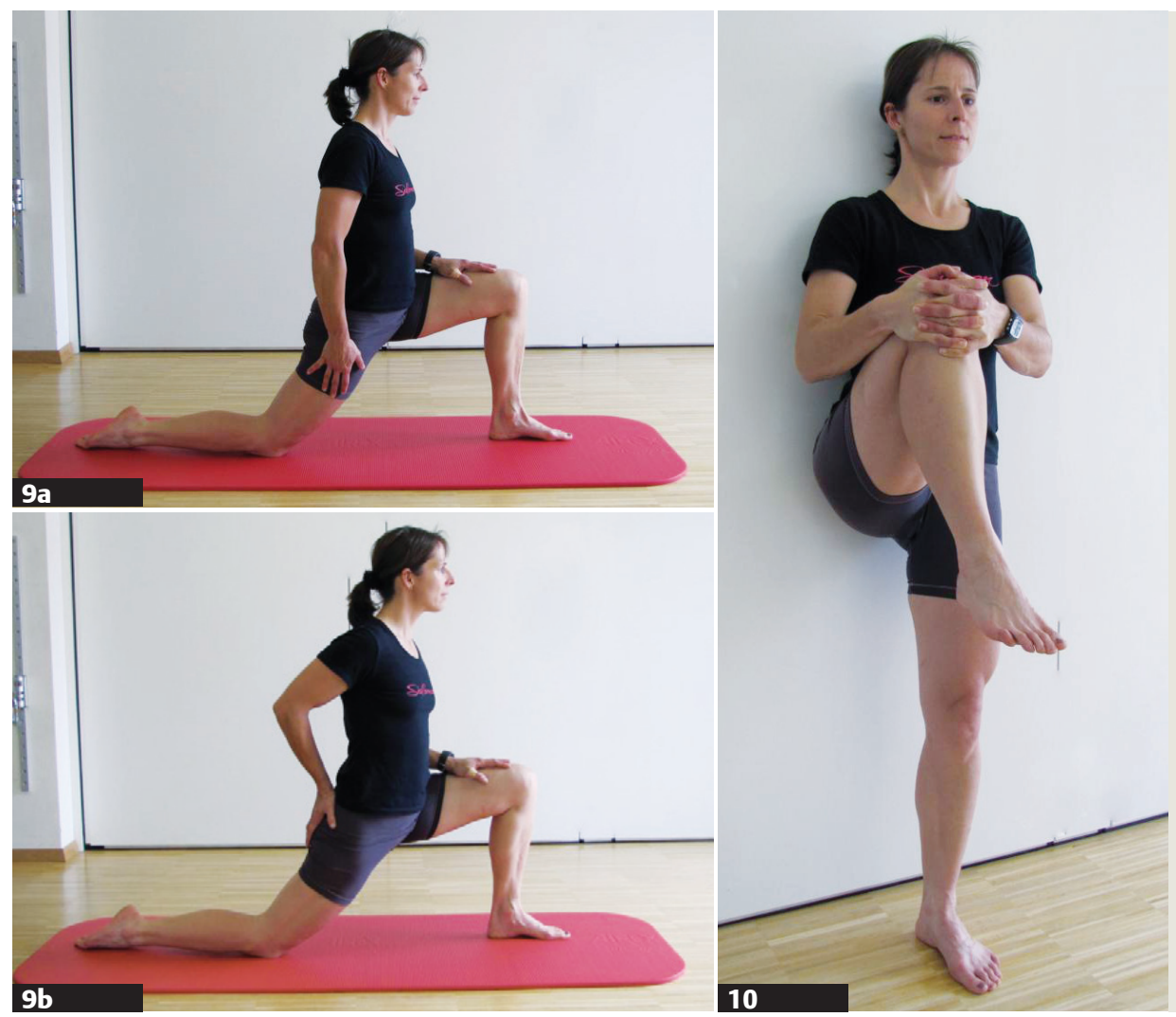

Abb. 9a u. b Repetierte Rotation des rechten Iliums im Einbeinkniestand. a Nach anterior. b Zusätzlich mit Überdruck durch die Patientin.

Abb. 10 Repetierte Rotation des rechten Iliums im Einbeinstand nach posterior.
Die Rotation des Iliums nach posterior erfüllt klar die Kriterien einer Directional Preference. „Alle zwei bis drei Stunden zehn repetierte Rotationen des rechten Iliums nach posterior“ lautet die neue Trainingsanleitung bis zum nächsten Besuch.

\section{Besuch 3}

Nach zehn Tagen stellt sich die Patientin wieder in der Praxis vor. Sie hat die Übungen sehr regelmäßig durchgeführt. Knie- und Oberschenkelschmerz sind seit dem letzten Besuch nicht mehr aufgetreten. Mehrstündige Mountainbiketouren hat sie sehr gut überstanden. Beim Berglauftraining hat sie den Gesäßschmerz noch gespürt, musste aber das Training nicht einschränken.

Die patientenspezifischen funktionellen Outcomes am Behandlungstag 3:

1. Berglauf: 3;

2. Intensiv Mountainbike fahren: 4;

3. Schwere körperliche Arbeit - z. B. Harken bei der Gartenarbeit: 2; Gartenarbeit hat sich die Patientin noch nicht zugetraut.
Repetierte Testbewegungen produzierten nach einigen Seitgleitbewegungen nach links den bekannten Gesäßschmerz mit leichter Intensität (VAS 2).

Um den Effekt von passiver Bewegung zu evaluieren, führt der Therapeut etwa 20 Mal eine endgradige, passive Rotation des Iliums nach posterior in Seitenlage durch ( $\mathbf{A b b} \mathbf{\text { b }} \mathbf{1 1})$.

Bei den anschließenden repetierten Seitgleitbewegungen kann die Patientin keinen Gesäßschmerz provozieren.

Die regelmäßige Rotation des rechten Iliums nach posterior bleibt weiterhin die Übung für das Eigentraining.

\section{Besuch 4}

14 Tage später folgt der nächste Besuch der Patientin. Sie hat weiterhin regelmäßig geübt. Bereits zehn Tage zuvor hat sie zwei Stunden ohne Beschwerden im Garten gearbeitet.

Am Wochenende vor dem Besuch beim Therapeuten hat sie zum ersten Mal seit Jahren wieder an einem Berglauf teilgenommen ( Abb. 12). Abgesehen von ordentlicher Erschöpfung an-

\section{DIRECTIONAL PREFERENCE}

Im McKenzie-Konzept der Mechanischen Diagnose und Therapie kommt der „Directional Preference“ eine besondere Bedeutung zu. Der Begriff beschreibt die Bewegungsrichtung oder Haltungsstrategie, welche Symptome reduziert, eliminiert oder zentralisiert und / oder die Beweglichkeit verbessert [15]. Auch das Klassifikationssystem „Treatment-Based-Classification (TBC)“ nach Delitto
[3-5, 7] nutzt „Directional Preference“ zur Subgruppenklassifikation. Während aber Delitto und seine Kollegen $[3-5,7]$ diese Richtungspräferenz nur bei Patienten mit Wirbelsäulenbeschwerden als Kriterium einsetzen, hilft das Auffinden einer Directional Preference im MDT-Konzept, auch Patienten mit Extremitätenbeschwerden klinisch einzuteilen [1, 8, 13, 14]. 

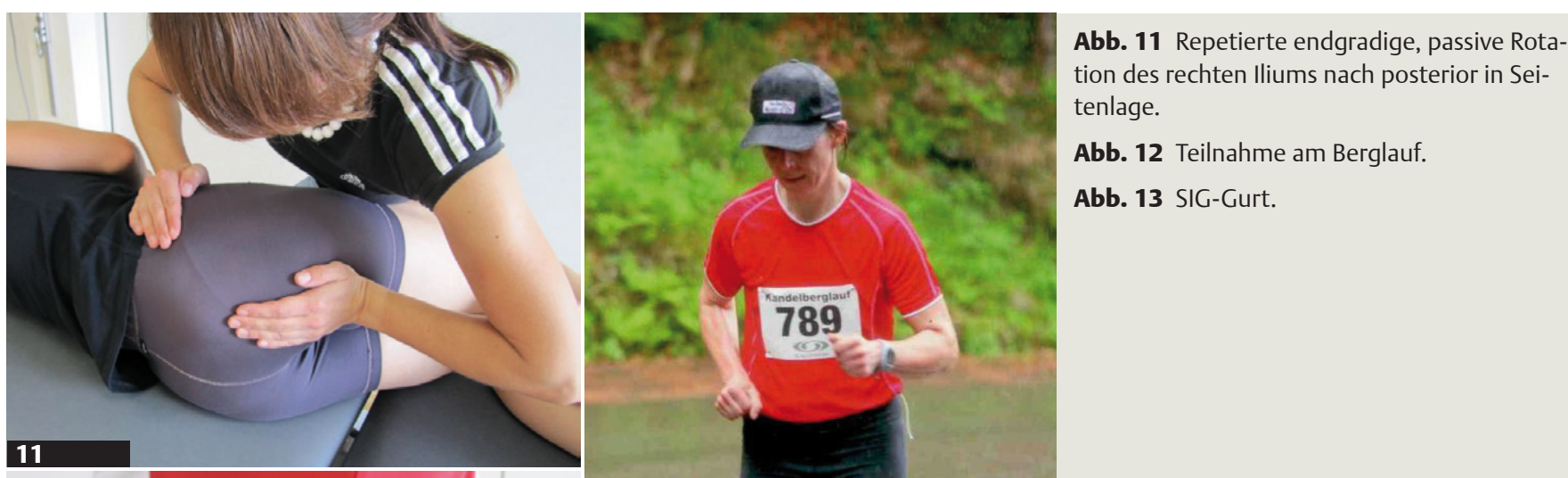
tion des rechten lliums nach posterior in Seitenlage.

11

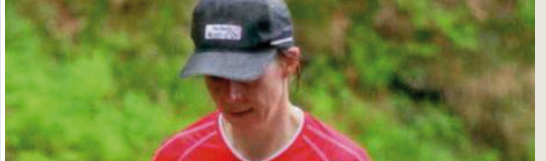

Abb. 12 Teilnahme am Berglauf.

Abb. 13 SIG-Gurt.
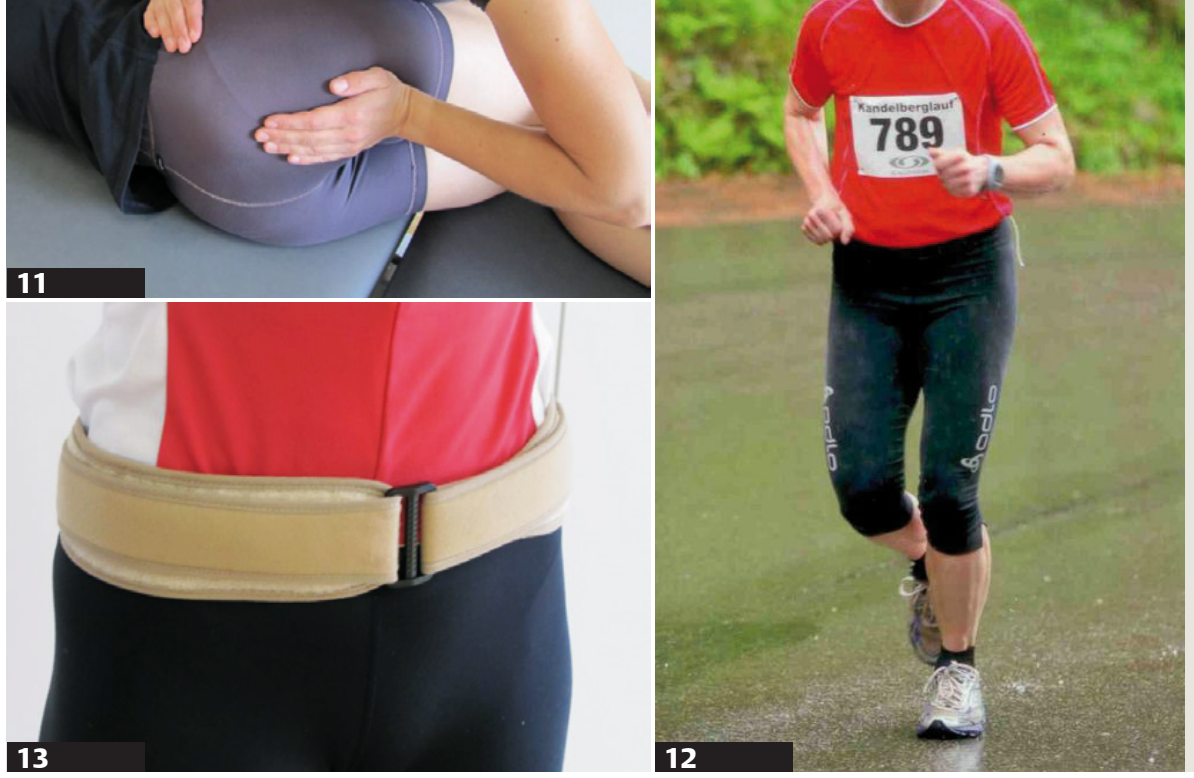

gesichts der ungewohnten Höhendifferenz (960 Höhenmeter bei $12 \mathrm{~km}$ Länge) hat sie weder während noch nach dem Lauf Beschwerden.

Die patientenspezifischen funktionellen Outcomes am Behandlungstag 4:

1. Berglauf: 4;

2. Intensiv Mountainbike fahren: 4;

3. Schwere körperliche Arbeit - zum Beispiel Harken bei Gartenarbeit: 4 .

Die abschließende klinische Untersuchung zeigt keinerlei Auffälligkeiten. Die Behandlung ist nach vier Terminen abgeschlossen.

\section{Kontakt nach zwei Monaten}

Die Patientin hat mittlerweile an einem weiteren Berglauf teilgenommen und dabei sogar in ihrer Altersklasse gewonnen. Längere Läufe bis zu 1,40 Stunden gehen problemlos.

Die Frequenz der Übungen hat sie mittlerweile reduziert, meistens einmal täglich. Wenn sie die Übungen mehrere Tage nicht macht und sich dann stärker körperlich belastet, spürt sie leichte Symptome tieflumbal rechts. Die bekannte Eigenübung hilft aber sofort.

Nach einer fünf Jahre dauernden Vorgeschichte ist sie nun schon seit zehn Wochen völlig beschwerdefrei und hat keinerlei Einschränkungen im Alltag und Sport.

\section{Studienergebnisse 1:1 übertragen?}

Obwohl laut aktueller wissenschaftlicher Evidenz sechs negative SIG-Tests dieses Gelenk als Schmerzursache ausschließen sollen [10], konnte sich die Patientin mit repetierten Bewegungen im Sinne einer Rotation des rechten Iliums nach posterior erfolgreich selbst behandeln.

Der Autor setzt das von Laslett beschriebene Vorgehen seit mehr als zehn Jahren in der täglichen Praxis bei orthopädischen und sporttraumatologischen Patienten ein. Bei vielen Patienten, die schließlich erfolgreich mit - auf das SIG zielender - mechanischer Therapie behandelt werden können, sind drei oder mehr Provokationstests positiv. Allerdings ist oftmals auch kein einziger Provokationstest positiv, doch in Untersuchung oder Probebehandlung zeigt sich trotzdem eine „Directional Preference“. Oder der Patient wird durch zeitlich begrenztes Tragen eines SIG-Gurts dauerhaft beschwerdefrei ( $\bullet$ Abb. 13).

Zurück zur eingangs gestellten Patientenfrage „Machen mich Deine Studien gesund?“ Therapeuten führt das eher zu der Frage: „Inwieweit kann ich die Ergebnisse und Schlussfolgerungen der Studien auf meinen klinischen Alltag übertragen?“ Verfahren, die irgendwann einmal zum Referenzstandard in der Diagnostik einer Pathologie werden, haben ihre Validität oft bei einer sehr speziellen Patientenklientel bewiesen. SIG-Infiltrationen lassen nur Patienten mit einem enormen Leidensdruck und hohem Invalidisierungsgrad über sich ergehen.

Mark Laslett hat mit seiner Forschung einen Quantensprung in der Diagnostik und Therapie von SIG-Problemen bewirkt. Dank 
ihm gehören nicht reliable Palpations- und Inspektionstests mit einem Validitätsgrad von 0 zusehends der Vergangenheit an.

Klinisch tätige Therapeuten sollten aber Lasletts Schlussfolgerungen nicht 1:1 übertragen. Nicht jeder Therapeut sieht in seiner Praxis regelmäßig LWS-/SIG-Patienten, die hochgradig chronifiziert, in psychometrischen Tests auffällig (Zung Depression Index Questionnaire, MSPQ = Modified Somatic Perception Questionnaire, DRAM = Distress Risk Assessment Method) und langzeitarbeitsunfähig sind. Das sind aber exakt die Patienten, die in Lasletts Studien zur Strukturdiagnose untersucht wurden [9-11].

\section{FAZIT}

Das Vorgehen, bei Patienten mit Beschwerden im Bereich LWS-

Becken-Bein zuerst die LWS als Schmerzursache auszuschließen, dann das Hüftgelenk zu untersuchen und schließlich reliable Schmerzprovokationstests anzuwenden, findet in der Fachwelt breite Zustimmung. Zu Recht!

Dogmatische Schlussfolgerungen sind allerdings fehl am Platz. Das vorliegende Fallbeispiel zeigt zum einen den pragmatischen Umgang mit diesem Verfahren. Zum anderen verdeutlicht es den klinischen Wert einer Untersuchung, die auf Bewegungstests und deren symptomatischen und mechanischen Antworten beruht.

Letztendlich zählt, den Patienten in eine Subgruppe einzuteilen, deren definierte Managementstrategie ihn bleibend verbessert. Provokativ, aber legitim ist die Frage: Hat die vorgestellte Patientin denn überhaupt ihr SIG behandelt? War es vielleicht das Hüftgelenk, das reagiert hat? Oder irgendeine periartikuläre Struktur?

Für den Therapeuten hat es gereicht, die Rotation des rechten Iliums nach posterior als Directional Preference auszumachen. Die Patientin war einfach nur froh, eine Übung zu haben, die hilft.

Die Literatur finden Sie online unter thieme connect: www.thieme-connect.de/ejournals/toc/manuelletherapie

\section{AUTOR}

Georg Supp, PT MT DipMDT

Georg Supp ist seit 1992 Physiotherapeut, Miteigentümer des Therapiezentrums PULZ in Freiburg und Senior Instructor des McKenzie Institute International. Als Mitglied des Board of Directors der International MDT Research Foundation setzt er sich für physiotherapeutische Forschungsprojekte ein.

PULZ

Rieselfeldallee 12

79111 Freiburg

georg.supp@pulz-freiburg.de

\section{BIBLIOGRAFIE}

DOI 10.1055/s-0032-1304756

manuelletherapie 2012; 16: 25-30

(c) Georg Thieme Verlag KG

Stuttgart · New York · ISSN 1433-2671 